\title{
A 1-Norbornene Adduct of a Transition-Metal, and an Electrochemical Study of the Bridgehead Olefin Complex
}

\author{
Gavin D. Jones and David A. Vicic* \\ Department of Chemistry and Biochemistry, University of Arkansas, Fayetteville, AR 72701
}

General Considerations. All manipulations were performed using standard Schlenk techniques or in a nitrogen-filled dry box, unless otherwise noted. Solvents were distilled from $\mathrm{Na}$ /benzophenone or $\mathrm{CaH}_{2}$. All reagents were used as received from commercial vendors unless otherwise noted. Aluminum oxide (activated, neutral, Brockmann I, 150 mesh) was dried at 200 ${ }^{\circ} \mathrm{C}$ under vacuum for two days prior to use. Elemental analyses were performed by Desert Analytics. ${ }^{1} \mathrm{H}$ NMR spectra were recorded at ambient temperature (unless otherwise noted) on a Bruker Avance $300 \mathrm{MHz}$ spectrometer and referenced to residual proton solvent peaks. ${ }^{31} \mathrm{P}\left\{{ }^{1} \mathrm{H}\right\}$ spectra were recorded on the Bruker Avance spectrometer operating at $121.44 \mathrm{MHz}$ and referenced to an $85 \%$ phosphoric acid external standard set to $0 \mathrm{ppm}$. A Rigaku MSC Mercury/AFC8 diffractometer was used for X-ray structural determinations. The compounds $\left(\mathrm{PCy}_{3}\right)_{2} \mathrm{Ni}\left(\eta^{2}\right.$-ethylene $),{ }^{1} \quad\left(\mathrm{PCy}_{3}\right)_{2} \mathrm{Ni}\left(\eta^{2}\right.$-1-hexene $),{ }^{2},\left(\mathrm{PCy}_{3}\right)_{2} \mathrm{NiCl}_{2},{ }^{3}$ 1-chloronorbornane, ${ }^{4}$ and $\left[\mathrm{Fe}(\mathrm{bpy})_{3}\right]\left(\mathrm{PF}_{6}\right)_{3},{ }^{5}$ were all prepared by previously published procedures.

Cyclic Voltammetry Measurements. Cyclic voltammetry was performed in predried solutions of $3 \mathrm{mM} \mathrm{Ni}$ complex in $0.1 \mathrm{M} \mathrm{TBAPF}_{6} / \mathrm{THF}\left(\mathrm{TBAPF}_{6}=\right.$ tetrabutylammonium hexafluorophosphate). The $\mathrm{TBAPF}_{6}$ was recrystallized from $\mathrm{THF} /$ pentane and dried on high vacuum manifold overnight). Measurements were performed at a scan rate of $10 \mathrm{mV} / \mathrm{sec}$ under an argon atmosphere. A platinum disk $(2.0 \mathrm{~mm}$ diameter, $\mathrm{CH}$ Instruments, Inc.) and a platinum flag were served as the working electrode and the counter electrode, respectively. A silver wire in a $0.01 \mathrm{M} \mathrm{AgNO}_{3}$ solution of DMF (anhydrous) served as non-aqueous reference electrode which was separated from the test solution by a fine glass frit. A $\mathrm{CH}$ Instrument, Inc. model CHI650A potentiostat and CHI650A personal computer software controlled the electrochemical experiments. The non-aqueous reference electrode kit was purchased from Bioanalytical Systems, Inc. model \#MF-2062.

Preparation of 1-norbornyl lithium: Procedure modified from previously reported method. ${ }^{6}$ All manipulations were performed under an argon atmosphere, unless otherwise noted. A twoneck $250 \mathrm{~mL}$ RBF was charged with 1-chloronorbornane $(4.83 \mathrm{~g}, 0.0369 \mathrm{~mol})$ in pentane $(125$ $\mathrm{mL})$. To that clear and colorless solution, Li powder $(0.531 \mathrm{~g}, 0.0769 \mathrm{~mol})$ was added. Solution refluxed under argon using Schlenk techniques for 1.25 hours. The solvents were removed under vacuum yielding a purple solid. In the glove box the purple solution was filtered through a glass fritted funnel with pentane collected the unreacted lithium and lithium chloride. The clear and colorless filtrate was titrated using salicylaldehyde phenylhydrazone. ${ }^{7}$ 1-norbornyl lithium was stored in a freezer at $-30{ }^{\circ} \mathrm{C}$. 
Preparation of $\left(\mathbf{P C y}_{3}\right)_{2} \mathbf{N i}\left(\eta^{2}\right.$-1-norbornene) (2): A $200 \mathrm{~mL}$ RBF equipped with a stir bar was charged with a slurry of $\left(\mathrm{PCy}_{3}\right)_{2} \mathrm{NiCl}_{2}(581 \mathrm{mg}, 0.841 \mathrm{mmol})$ and pentane $(100 \mathrm{~mL})$. To that red slurry, 1-norbornyl lithium (15.3 mL, $1.68 \mathrm{mmol}, 0.11 \mathrm{M}$ in pentane) was added via syringe and stirred for 10 minutes at which time the reaction became orangish-brown. The solution was passed over a $1 \mathrm{~cm}$ pad of alumina and thorough rinsing with pentane yielded an orange filtrate. The solvents were removed from the filtrate on high vacuum manifold. The crude product is further purified by extraction with pentane and passed over a $1 \mathrm{~cm}$ pad of alumina and thorough rinsing with pentane yielded an orange filtrate. Solvents were removed again on high vacuum line yielding $398 \mathrm{mg}(66 \%)$ of an orange solid. X-ray quality crystals were grown from a concentrated solution of $\mathbf{2}$ in pentane filtered through a glass wool plugged pipet and stored in a glass vial capped for 3 days in a freezer $\left(-30{ }^{\circ} \mathrm{C}\right) .{ }^{31} \mathrm{P}\left\{{ }^{1} \mathrm{H}\right\} \mathrm{NMR}\left(\mathrm{C}_{6} \mathrm{D}_{6}, 30^{\circ} \mathrm{C}, 121.5 \mathrm{MHz}\right): \delta$ $44.14(\mathrm{~d}, J=20.74 \mathrm{~Hz}), 39.63(\mathrm{~d}, J=20.76 \mathrm{~Hz})$. Anal. Calc'd (found) for $\mathrm{C}_{43} \mathrm{H}_{76} \mathrm{NiP}_{2}: 72.36$ (72.12) \%C; $10.73(10.49) \% \mathrm{H}$.

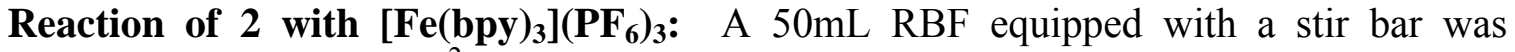
charged with $\left(\mathrm{PCy}_{3}\right)_{2} \mathrm{Ni}\left(\eta^{2}\right.$-norbornene) $(289 \mathrm{mg}, 0.405 \mathrm{mmol})$ and furan $(20 \mathrm{~mL})$. To that orange solution, $\left[\mathrm{Fe}(\mathrm{bpy})_{3}\right]\left(\mathrm{PF}_{6}\right)_{3}(385 \mathrm{mg}, 0.401 \mathrm{mmol})$ was added and stirred giving an immediate dark green slurry. The reaction was stirred for $15 \mathrm{hr}$ giving a dark red precipitate and faint yellow, clear solution. The contents of the reaction were separated using a silica gel column eluting with $\mathrm{CH}_{2} \mathrm{Cl}_{2}$. Evaporation of solvents yielded $31 \mathrm{mg}(81 \%)$ of dinorbornyl (characterized by X-ray and GC-MS).

Preparation of (dippe)NiMe: To a stirred suspension of (dippe) $\mathrm{NiCl}_{2}{ }^{8}(2.0 \mathrm{~g}, 5 \mathrm{mmol})$ in ether $(200 \mathrm{~mL})$ at $-78^{\circ} \mathrm{C}$ was added methyl lithium $(6.4 \mathrm{~mL}$ of a $1.6 \mathrm{M}$ solution in ether). The suspension was warmed to room temperature and stirred for $0.5 \mathrm{~h}$. The suspension was then filtered over a small pad of alumina, and the solvent was removed under vacuum. The residue was then extracted with pentane, and the solution was cooled to $-78{ }^{\circ} \mathrm{C}$, during which time yellow crystals precipitated. The crystals were filtered, washed with cold pentane, and dried. Yield: $760 \mathrm{mg}(43 \%)$. ${ }^{1} \mathrm{H} \mathrm{NMR}\left(\mathrm{C}_{6} \mathrm{D}_{6}, 30{ }^{\circ} \mathrm{C}, 300 \mathrm{MHz}\right): \delta 1.96$ (oct, J = $\left.6.95 \mathrm{~Hz}, 4 \mathrm{H}\right), 1.13$ $(\mathrm{dd}, \mathrm{J}=15.0,6.95 \mathrm{~Hz}, 12 \mathrm{H}), 1.02(\mathrm{~d}, \mathrm{~J}=10.25 \mathrm{~Hz}, 4 \mathrm{H}), 0.87$ (dd, J = 12.08, $6.95 \mathrm{~Hz}, 12 \mathrm{H}), 0.55$

$(\mathrm{dd}, \mathrm{J}=9.15,4.39 \mathrm{~Hz}, 6 \mathrm{H}) ;{ }^{31} \mathrm{P}\left\{{ }^{1} \mathrm{H}\right\} \mathrm{NMR}\left(\mathrm{C}_{6} \mathrm{D}_{6}, 30{ }^{\circ} \mathrm{C}, 121.5 \mathrm{MHz}\right): \delta 77.01$ (s). Full spectra are provided below. 
(dippe)Ni(CH3)2_1H_C6D6

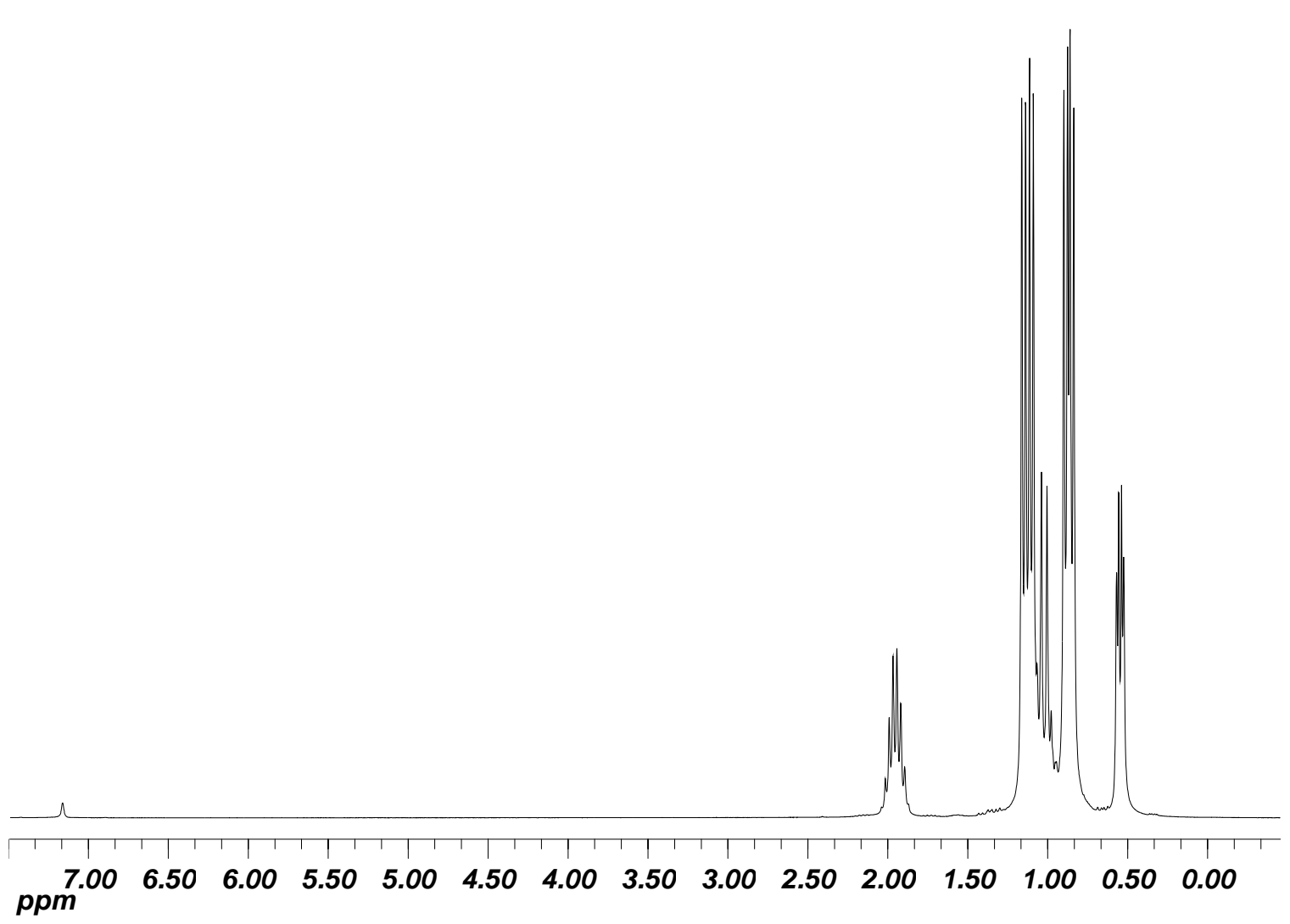

Figure 1S: ${ }^{1} \mathrm{H}$ NMR of (dippe)NiMe ${ }_{2}$ in $\mathrm{C}_{6} \mathrm{D}_{6}$ : 
(dippe)Ni(CH3)2_31 P_C6D6

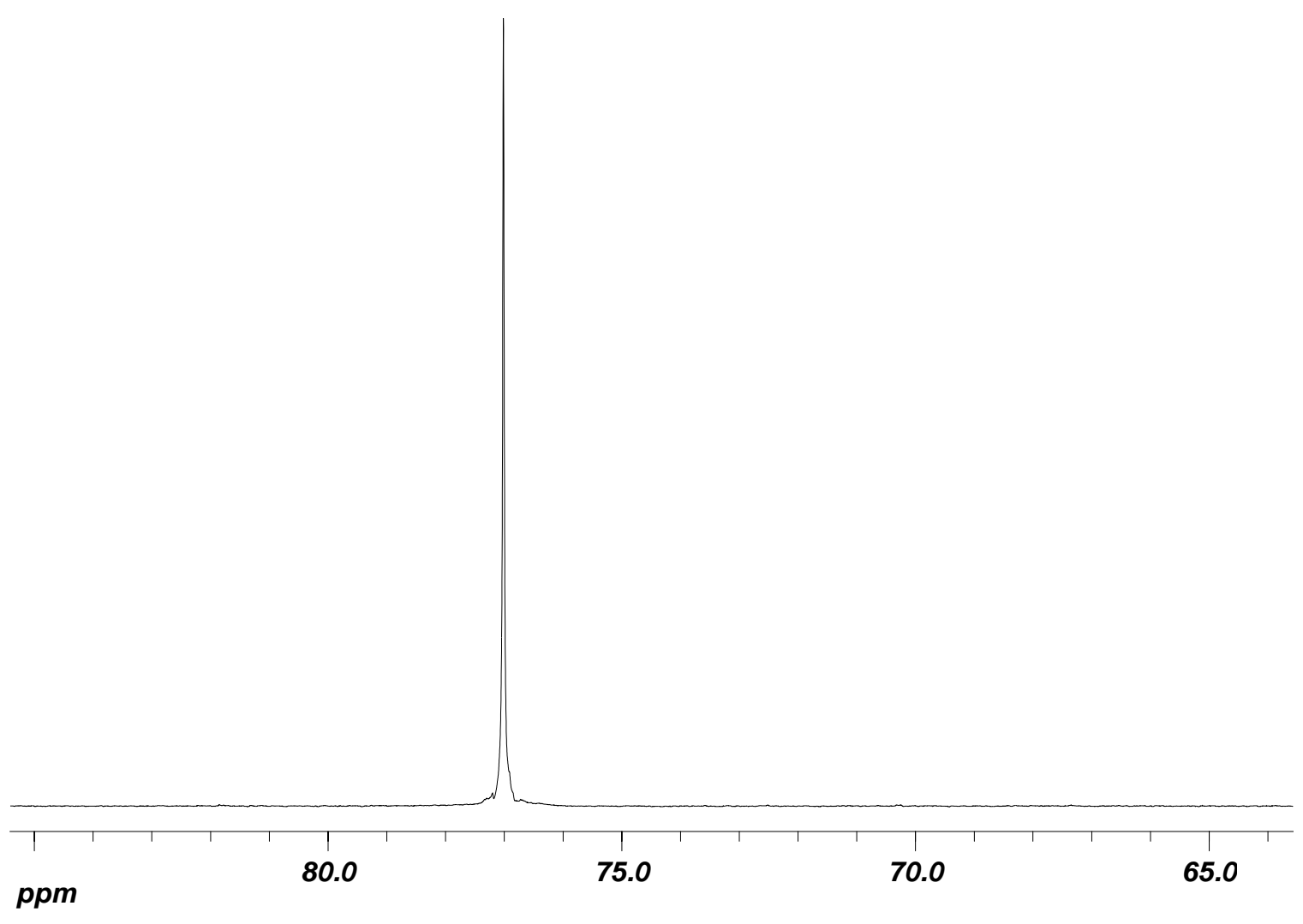

Figure $2 \mathrm{~S}:{ }^{31} \mathrm{P}\left\{{ }^{1} \mathrm{H}\right\}$ NMR of (dippe) $\mathrm{NiMe}_{2}$ in $\mathrm{C}_{6} \mathrm{D}_{6}$. 


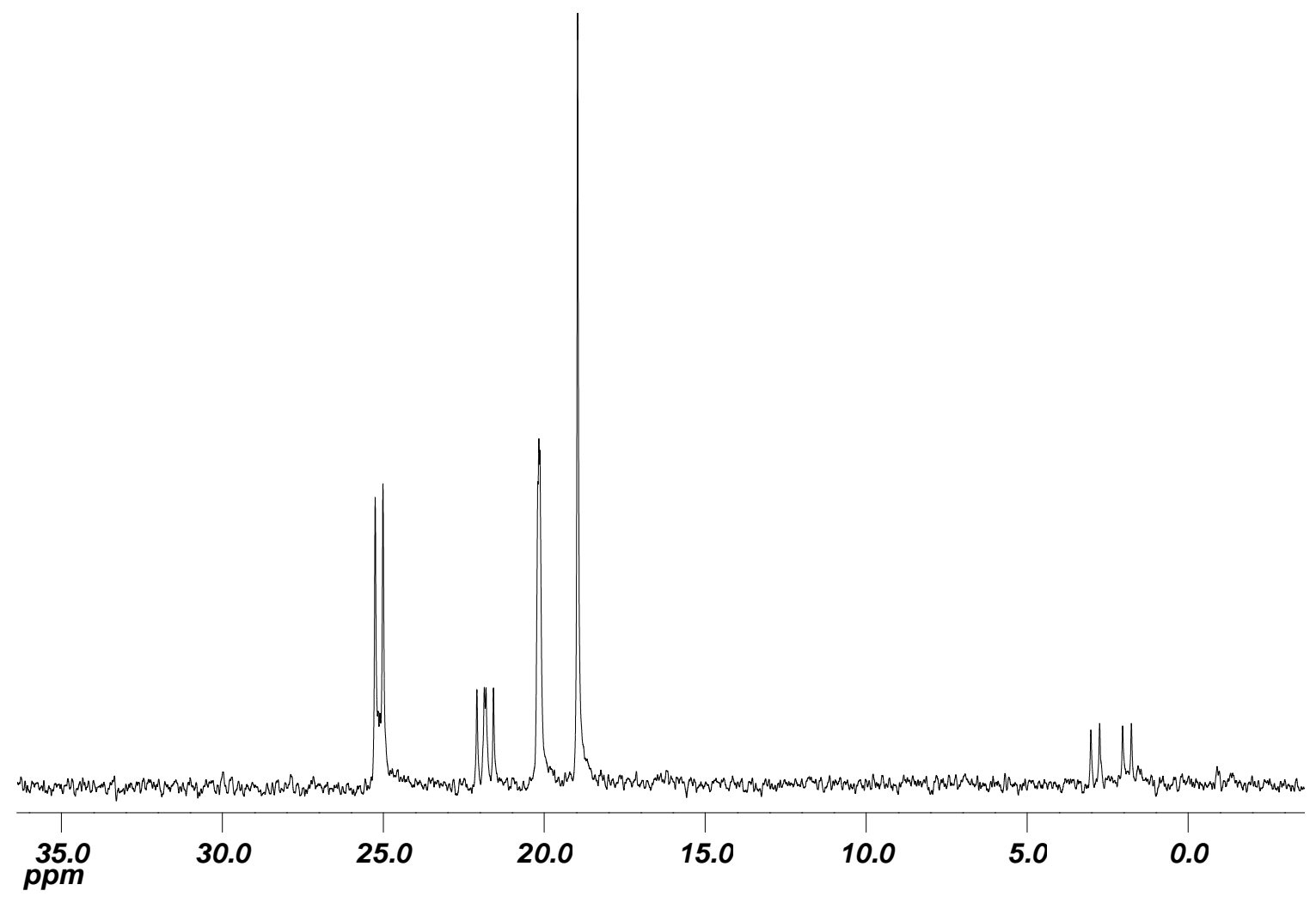

Figure 3S: ${ }^{13} \mathrm{C}\left\{{ }^{1} \mathrm{H}\right\}$ NMR of (dippe) NiMe 2 in $\mathrm{C}_{6} \mathrm{D}_{6}$.

1. Jolly, P. W.; Jonas, K. Inorganic Syntheses. 1974, 15, 29-31.

2. Musco, A.; Georgii, I.; Mann, B. E. Inorg. Chim. Acta. 1980, 45, L149-L150.

3. Stone, P. J.; Dori, Z. Inorganica Chimica Acta 1971, 5, 434-8.

4. Rieke, R. D.; Bales, S. E.; Hudnall, P. M.; Poindexter, G. S. Organic Syntheses 1980, 59, 85-86.

5. (a) Burstall, F. H.; Nyholm, R. S. Journal of the Chemical Society 1952, 3570-3579. Keene, F. R.; Salmon, D. J.; Walsh, J. L.; Abrufia, H. D.; Meyer, T. J. Inorg. Chem. 1980, 19, 1896-1903. (b) Substituted $\mathrm{HPF}_{6}$ with $\mathrm{NH}_{4} \mathrm{PF}_{6}$.

6. Grovenstein, Jr., E.; Singh, J.; Patel, B. B.; VanDerveer, D. Tetrahedron 1994, 50, 59715998.

7. Love, B. E.; Jones, E. G. J. Org. Chem. 1999, 64, 3755-3756.

8. $\quad$ Vicic, D. A.; Jones, W. D. J. Am. Chem. Soc. 1997, 119, 10857-10858. 
CV Data for 2:
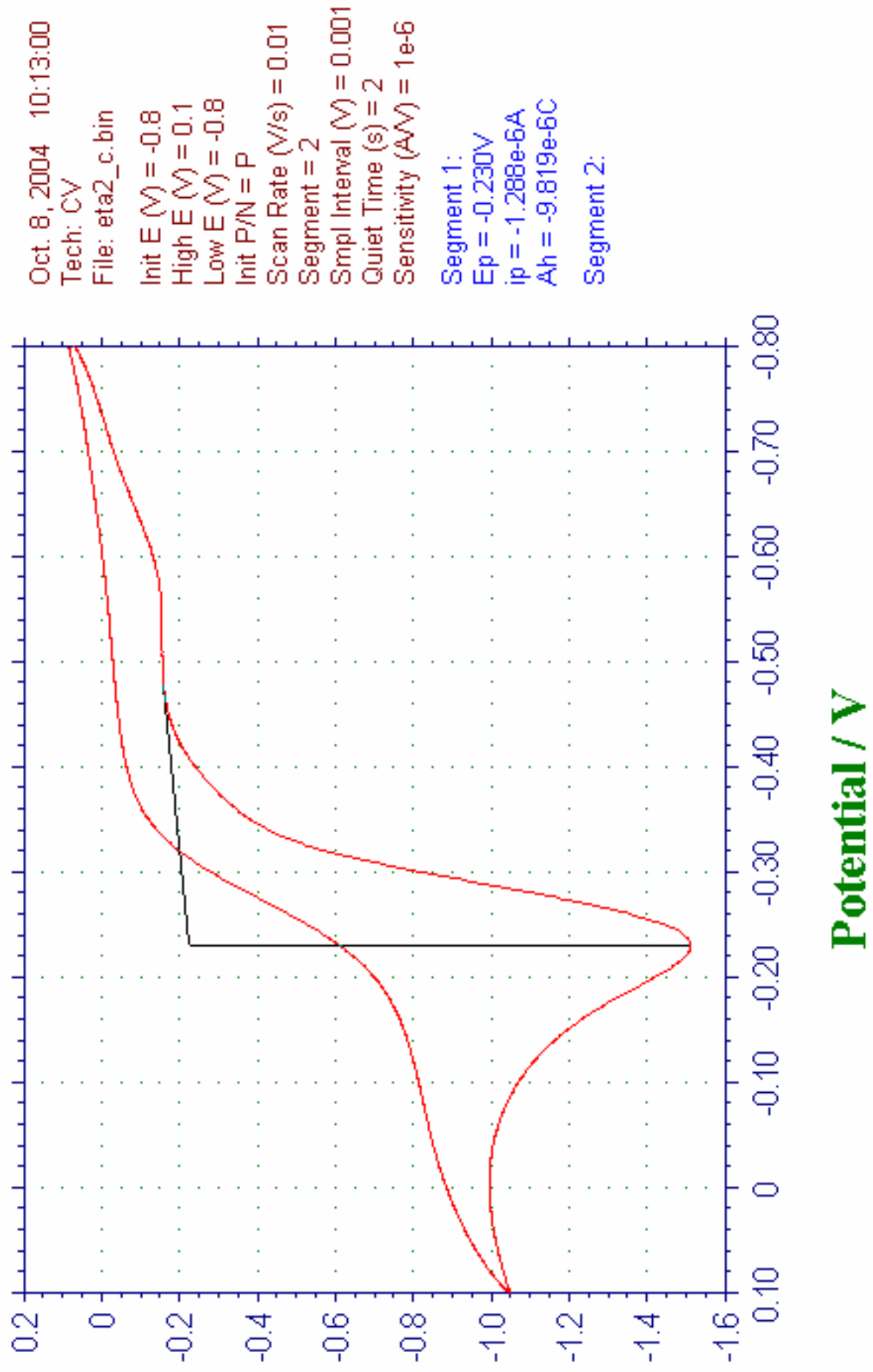

V9-əI / 1Uә.I.In 
$\mathrm{CV}$ Data for $\left(\mathrm{PCy}_{3}\right)_{2} \mathrm{Ni}\left(\eta^{2}\right.$-ethylene):
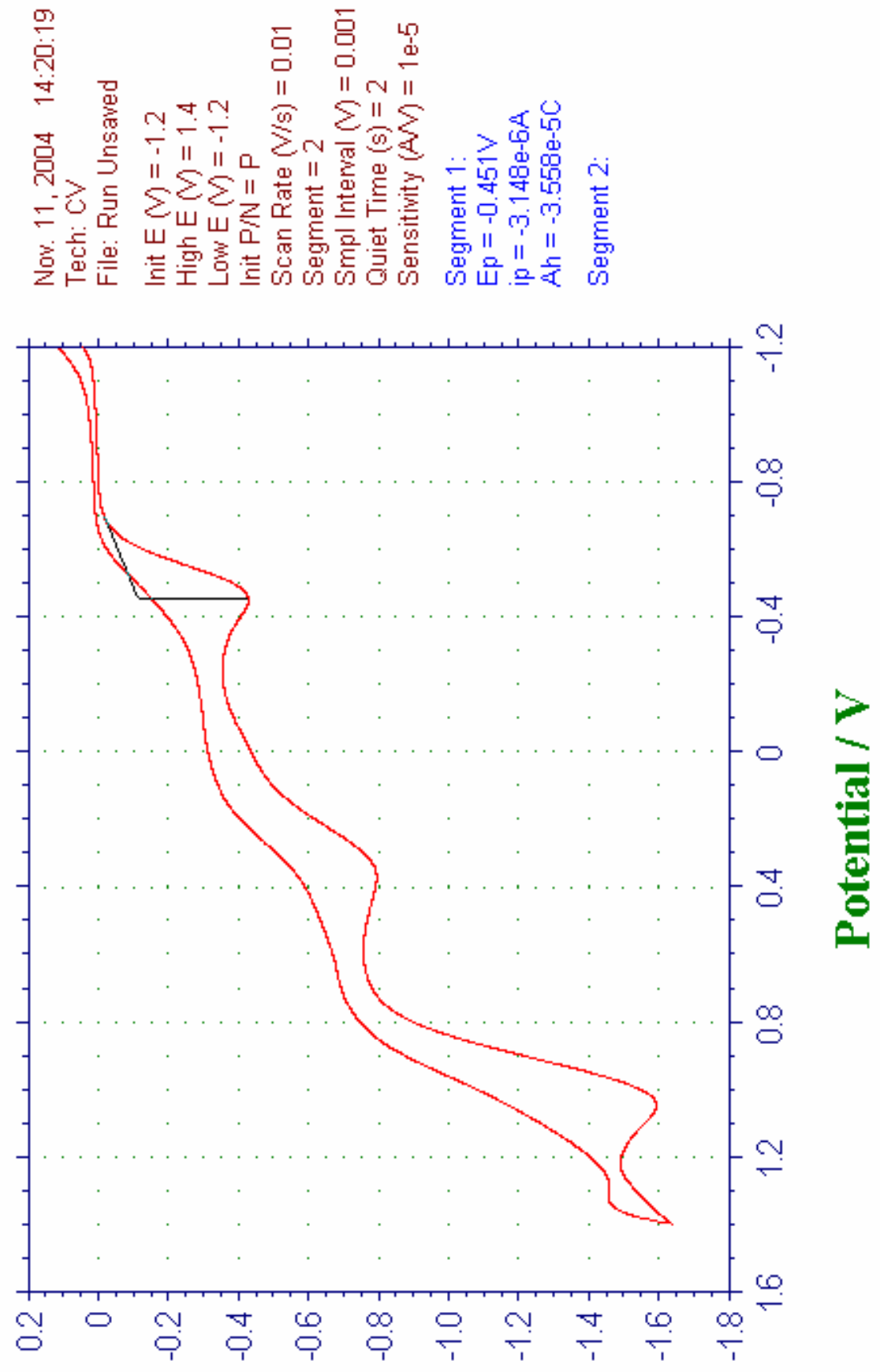

VS-o I / मUa.I.In 
CV Data for $\left(\mathrm{PCy}_{3}\right)_{2} \mathrm{Ni}\left(\eta^{2}-1\right.$-hexene $)$ :
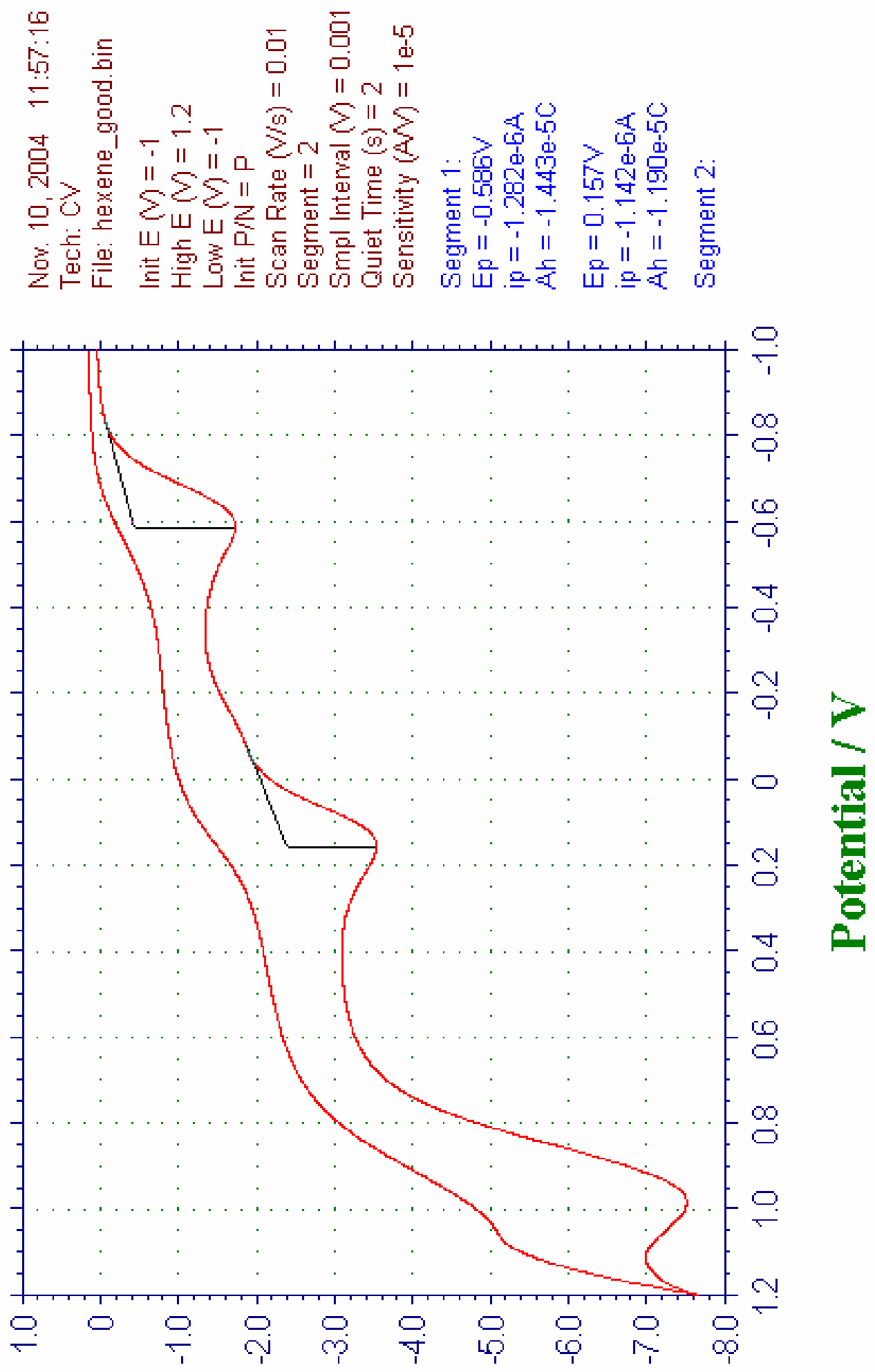

V9-əI / मUә.I.In 
$\mathrm{CV}$ data for (dippe) $\mathrm{NiMe}_{2}$ :

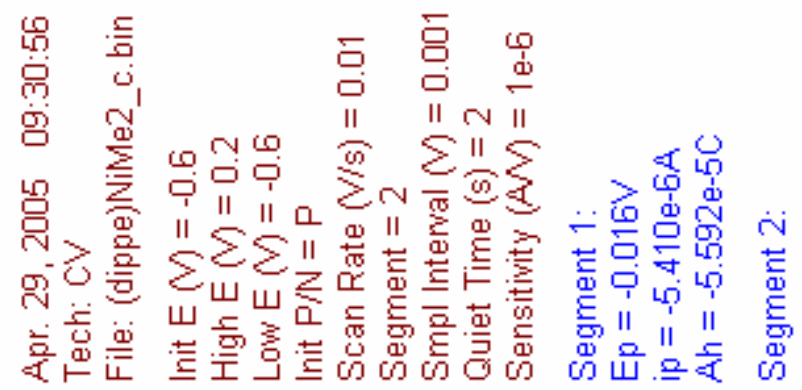

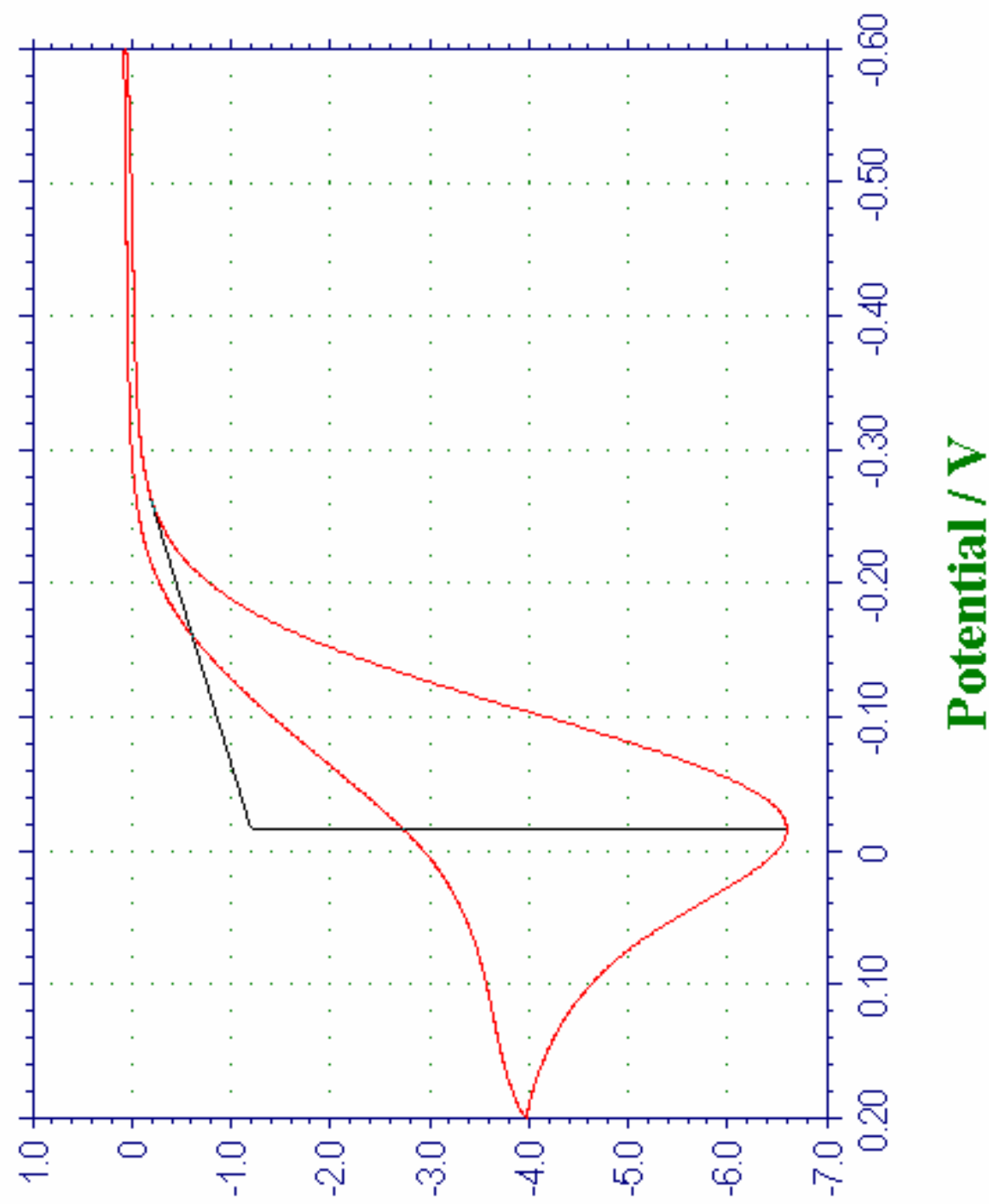

V9-๐ [ / 1Uว.I.In 
X-ray data for $2 \cdot$ pentane:
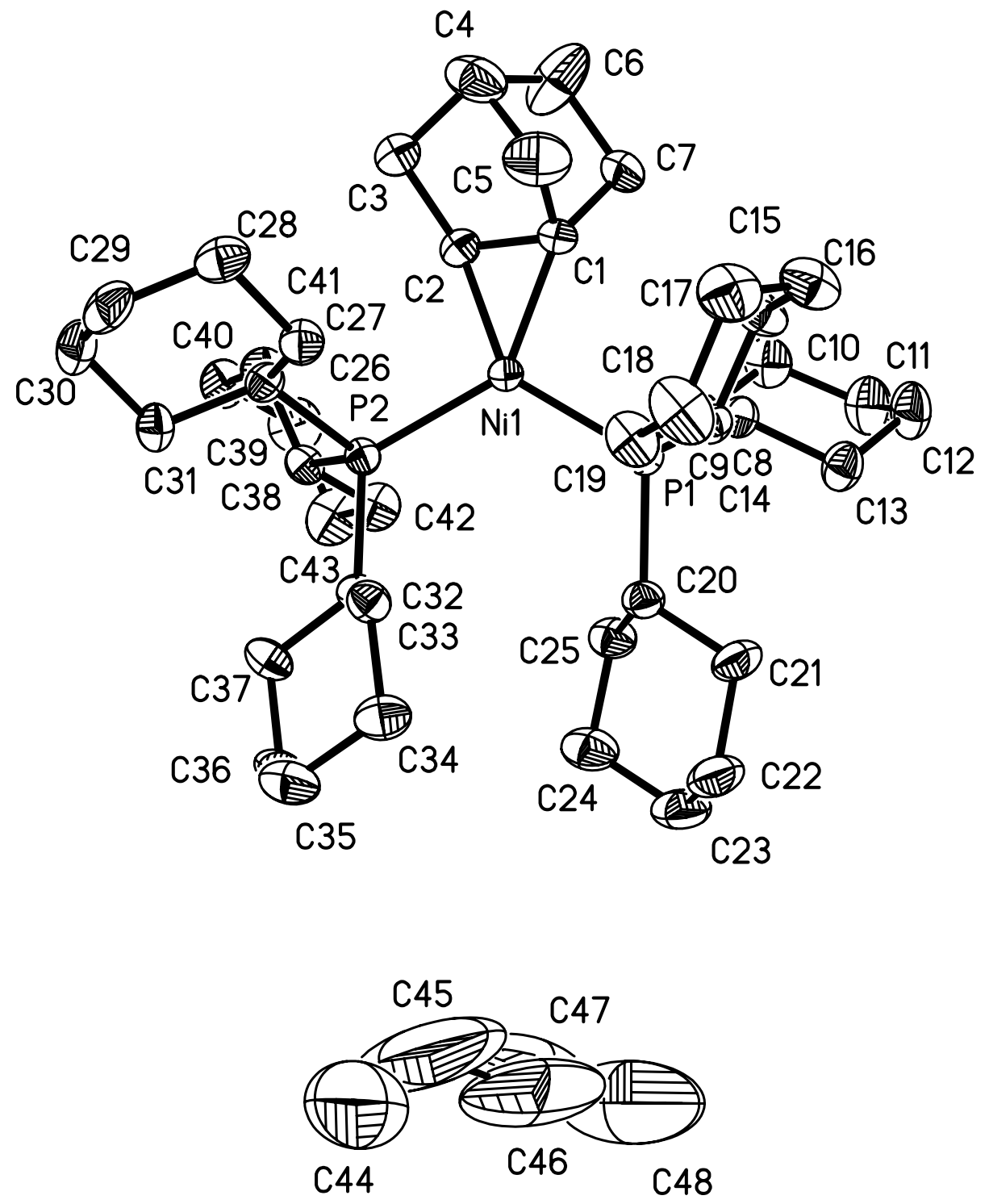
Table S1. Crystal data and structure refinement for Compound 2.(pentane).

\begin{tabular}{|c|c|}
\hline Identification code & Compound $2 \cdot($ pentane $)$ \\
\hline Empirical formula & $\mathrm{C} 48 \mathrm{H} 88 \mathrm{Ni} \mathrm{P} 2$ \\
\hline Formula weight & 785.83 \\
\hline Temperature & $173(2) \mathrm{K}$ \\
\hline Wavelength & $0.71070 \mathrm{~A}$ \\
\hline Crystal system, space group & monoclinic, $\mathrm{P} 21 / \mathrm{a}$ \\
\hline Unit cell dimensions & $\begin{array}{l}7.669(19) \text { A alpha }=90 \mathrm{deg} . \\
5.984(12) \text { A beta }=115.389(12) \text { deg. } \\
8.49(3) \text { A gamma }=90 \mathrm{deg} .\end{array}$ \\
\hline Volume & $4717(9) \quad A^{\wedge} 3$ \\
\hline Z, Calculated density & 4, $1.107 \mathrm{Mg} / \mathrm{m} \wedge 3$ \\
\hline Absorption coefficient & $\odot .509 \mathrm{~mm} \wedge-1$ \\
\hline$F(\odot \odot \odot)$ & 1736 \\
\hline Crystal size & $0.20 \times 0.20 \times 0.25 \mathrm{~mm}$ \\
\hline Theta range for data collection & 1.80 to $28.0 \odot \mathrm{deg}$. \\
\hline Limiting indices & $-23<=\mathrm{h}<=2 \odot, \quad-16<=\mathrm{k}<=21, \quad-13<=\mathrm{l}<=24$ \\
\hline Reflections collected / unique & $31713 / 11174[R($ int $)=0.0449]$ \\
\hline Completeness to theta $=28.00$ & $98 . \odot \%$ \\
\hline Refinement method & Full-matrix least-squares on $F^{\wedge} 2$ \\
\hline Data / restraints / parameters & $11174 / \odot / 463$ \\
\hline Goodness-of-fit on $F^{\wedge} 2$ & 1.109 \\
\hline Final $\mathrm{R}$ indices [I>2sigma(I)] & $\mathrm{R} 1=0.0777, \mathrm{WR} 2=0.1779$ \\
\hline $\mathrm{R}$ indices (all data) & $\mathrm{R} 1=0.1013, W R 2=0.1929$ \\
\hline Largest diff. peak and hole & 1.959 and -0.676 e. $A^{\wedge}-3$ \\
\hline
\end{tabular}


Table S2. Atomic coordinates $(x 10 \wedge 4)$ and equivalent isotropic displacement parameters $\left(A^{\wedge} 2 \times 10 \wedge 3\right)$ for Compound $2 \cdot$ (pentane). $\mathrm{U}(\mathrm{eq})$ is defined as one third of the trace of the orthogonalized Uij tensor.

\begin{tabular}{|c|c|c|c|c|}
\hline & $x$ & $y$ & z & $\mathrm{U}(\mathrm{eq})$ \\
\hline $\mathrm{Ni}(1)$ & $1558(1)$ & $3579(1)$ & $3138(1)$ & $24(1)$ \\
\hline$P(1)$ & $1619(1)$ & $3299(1)$ & $2007(1)$ & $24(1)$ \\
\hline$P(2)$ & 2078(1) & $4762(1)$ & $3741(1)$ & $23(1)$ \\
\hline$C(1)$ & $896(3)$ & $2632(3)$ & $3206(3)$ & $50(1)$ \\
\hline$C(2)$ & $1347(3)$ & $3030(3)$ & $3980(3)$ & $56(1)$ \\
\hline$c(3)$ & $743(4)$ & $3098(4)$ & $4371(4)$ & $80(2)$ \\
\hline$C(4)$ & $142(3)$ & $2427(3)$ & $3960(4)$ & $6 \odot(1)$ \\
\hline$C(5)$ & $31(3)$ & 2758 (3) & 3064 (3) & $63(1)$ \\
\hline$c(6)$ & $561(4)$ & $1607(4)$ & $3932(4)$ & $74(2)$ \\
\hline$C(7)$ & $926(4)$ & $1678(3)$ & $3325(3)$ & $60(1)$ \\
\hline$C(8)$ & $1945(2)$ & $2192(2)$ & $2022(2)$ & $29(1)$ \\
\hline$c(9)$ & $2698(2)$ & $1934(2)$ & $2792(2)$ & $35(1)$ \\
\hline$c(10)$ & $2790(3)$ & $981(2)$ & $2840(3)$ & $43(1)$ \\
\hline$C(11)$ & $2864(3)$ & $626(3)$ & 2106 (3) & $51(1)$ \\
\hline$C(12)$ & $2141(3)$ & $906(2)$ & $1342(3)$ & $48(1)$ \\
\hline$C(13)$ & $2052(3)$ & $1860(2)$ & $1294(2)$ & $38(1)$ \\
\hline$C(14)$ & $589(2)$ & $3329(2)$ & $1110(2)$ & $27(1)$ \\
\hline$C(15)$ & $-2(2)$ & $2611(2)$ & $1067(2)$ & $40(1)$ \\
\hline$C(16)$ & $-854(3)$ & $2712(3)$ & $356(3)$ & $46(1)$ \\
\hline$C(17)$ & $-1258(3)$ & $3538(3)$ & $357(3)$ & $52(1)$ \\
\hline$C(18)$ & $-679(3)$ & $4249(3)$ & $373(3)$ & $53(1)$ \\
\hline$C(19)$ & $175(2)$ & $4177(2)$ & $1083(3)$ & $40(1)$ \\
\hline$c(20)$ & $2288(2)$ & $3967(2)$ & $1678(2)$ & $30(1)$ \\
\hline$C(21)$ & $2205(3)$ & $3895(3)$ & $820(3)$ & $42(1)$ \\
\hline$C(22)$ & 2745 (3) & $4543(3)$ & $651(3)$ & $51(1)$ \\
\hline$c(23)$ & $3653(3)$ & $4487(3)$ & $1246(3)$ & $53(1)$ \\
\hline$C(24)$ & $3743(3)$ & $4567(3)$ & $2107(3)$ & $46(1)$ \\
\hline$C(25)$ & $3213(2)$ & $3900(2)$ & $2269(3)$ & $36(1)$ \\
\hline$C(26)$ & $1298(2)$ & $5266(2)$ & $4 \odot 26(2)$ & $26(1)$ \\
\hline$C(27)$ & $406(2)$ & $5279(2)$ & $3348(2)$ & $32(1)$ \\
\hline$C(28)$ & $-231(2)$ & $5541(2)$ & $3662(3)$ & $39(1)$ \\
\hline$C(29)$ & $2(3)$ & $6388(2)$ & $4084(3)$ & $43(1)$ \\
\hline$C(30)$ & $877(3)$ & $6373(2)$ & 4759 (3) & $42(1)$ \\
\hline$C(31)$ & $1525(2)$ & $6116(2)$ & $4453(2)$ & $33(1)$ \\
\hline$c(32)$ & $2431(2)$ & $5576(2)$ & $3217(2)$ & $27(1)$ \\
\hline$C(33)$ & $1680(2)$ & $5969(2)$ & $2528(2)$ & $30(1)$ \\
\hline$C(34)$ & $1948(3)$ & $6530(2)$ & $2006(3)$ & $42(1)$ \\
\hline$C(35)$ & $2535(3)$ & $7214(3)$ & $2512(3)$ & $50(1)$ \\
\hline$C(36)$ & $3287(3)$ & $6830(3)$ & $3189(3)$ & $45(1)$ \\
\hline$C(37)$ & $3043(2)$ & $6265(2)$ & $3718(2)$ & $36(1)$ \\
\hline$C(38)$ & $3004(2)$ & $4666(2)$ & $4723(2)$ & $28(1)$ \\
\hline$C(39)$ & $2818(2)$ & $4312(2)$ & $54 \odot 4(2)$ & $35(1)$ \\
\hline$C(40)$ & $3609(3)$ & $4239(3)$ & $6180(2)$ & $41(1)$ \\
\hline$C(41)$ & $4273(3)$ & $3712(3)$ & $6078(3)$ & $51(1)$ \\
\hline$C(42)$ & $4473(3)$ & $4066(3)$ & $5418(3)$ & $54(1)$ \\
\hline$c(43)$ & $3683(2)$ & $4144(3)$ & $4631(2)$ & $39(1)$ \\
\hline
\end{tabular}




$\begin{array}{lllll}C(44) & 4027(11) & 8245(10) & 1472(7) & 223(9) \\ C(45) & 4177(17) & 7441(13) & 1487(16) & 370(19) \\ C(46) & 4568(9) & 6906(11) & 1031(7) & 218(9) \\ C(47) & 5176(10) & 6493(9) & 1547(8) & 197(8) \\ C(48) & 5496(13) & 5951(9) & 1061(15) & 321(15)\end{array}$

Table S3. Bond lengths [A] and angles [deg] for Compound 2. (pentane).

\begin{tabular}{ll}
\hline & \\
$N i(1)-C(1)$ & $1.949(4)$ \\
$N i(1)-C(2)$ & $1.957(5)$ \\
$N i(1)-P(1)$ & $2.186(3)$ \\
$N i(1)-P(2)$ & $2.1878(16)$ \\
$P(1)-C(8)$ & $1.858(3)$ \\
$P(1)-C(14)$ & $1.864(4)$ \\
$P(1)-C(20)$ & $1.876(4)$ \\
$P(2)-C(38)$ & $1.857(4)$ \\
$P(2)-C(26)$ & $1.858(3)$ \\
$P(2)-C(32)$ & $1.881(4)$ \\
$C(1)-C(5)$ & $1.451(7)$ \\
$C(1)-C(2)$ & $1.454(6)$ \\
$C(1)-C(7)$ & $1.538(6)$ \\
$C(2)-C(3)$ & $1.527(6)$ \\
$C(3)-C(4)$ & $1.471(7)$ \\
$C(4)-C(6)$ & $1.518(6)$ \\
$C(4)-C(5)$ & $1.668(8)$ \\
$C(6)-C(7)$ & $1.519(7)$ \\
$C(8)-C(9)$ & $1.531(5)$ \\
$C(8)-C(13)$ & $1.531(5)$ \\
$C(9)-C(10)$ & $1.530(5)$ \\
$C(10)-C(11)$ & $1.528(6)$ \\
$C(11)-C(12)$ & $1.509(6)$ \\
$C(12)-C(13)$ & $1.531(5)$ \\
$C(14)-C(19)$ & $1.530(5)$ \\
$C(14)-C(15)$ & $1.531(5)$ \\
$C(15)-C(16)$ & $1.525(5)$ \\
$C(16)-C(17)$ & $1.502(6)$ \\
$C(17)-C(18)$ & $1.522(6)$ \\
$C(18)-C(19)$ & $1.521(6)$ \\
$C(20)-C(25)$ & $1.531(5)$ \\
$C(20)-C(21)$ & $1.534(6)$ \\
$C(21)-C(22)$ & $1.528(5)$ \\
$C(22)-C(23)$ & $1.509(7)$ \\
$C(23)-C(24)$ & $1.537(7)$ \\
$C(24)-C(25)$ & $1.531(5)$ \\
$C(26)-C(31)$ & $1.535(5)$ \\
$C(26)-C(27)$ & $1.537(5)$ \\
$C(27)-C(28)$ & $1.529(5)$ \\
$C(28)-C(29)$ & $1.528(6)$ \\
$C(29)-C(30)$ & $1.515(6)$ \\
$C(30)-C(31)$ & $1.535(5)$ \\
$C(32)-C(33)$ & $1.525(5)$ \\
$C(32)-C(37)$ & $1.543(5)$ \\
&
\end{tabular}




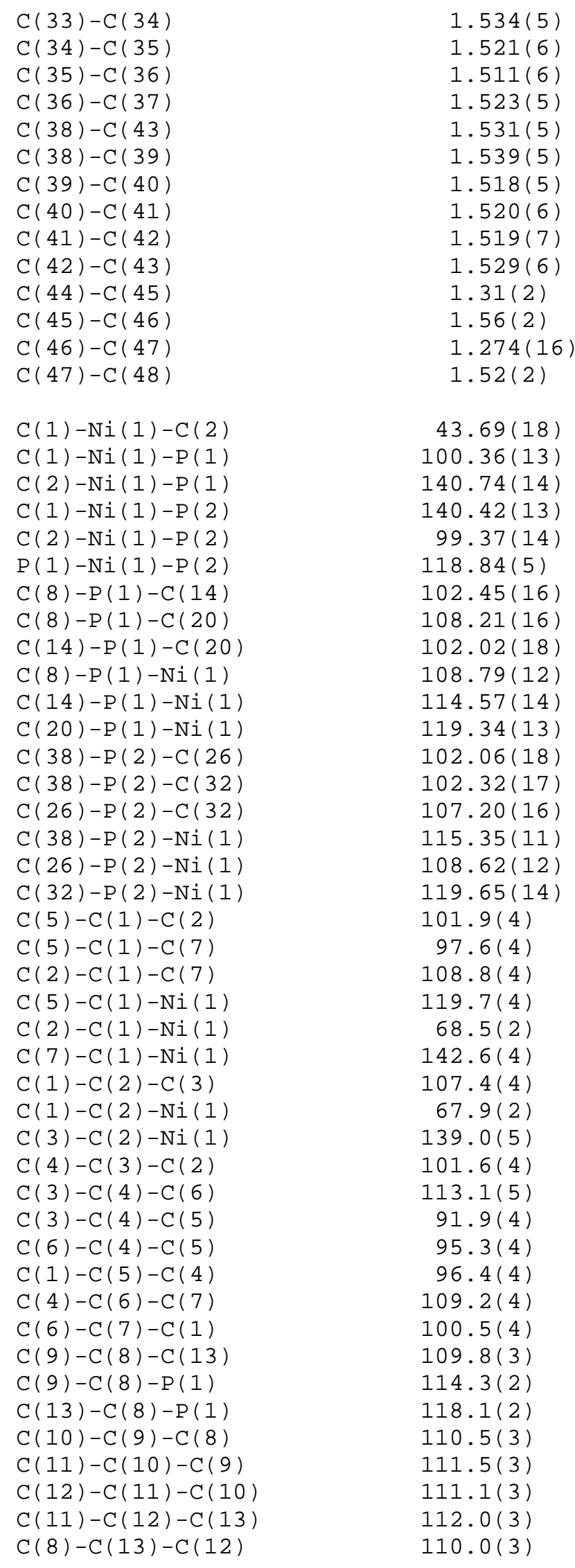




$\begin{array}{ll}C(19)-C(14)-C(15) & 110.9(3) \\ C(19)-C(14)-P(1) & 108.8(2) \\ C(15)-C(14)-P(1) & 114.3(2) \\ C(16)-C(15)-C(14) & 111.4(3) \\ C(17)-C(16)-C(15) & 112.8(4) \\ C(16)-C(17)-C(18) & 109.9(4) \\ C(19)-C(18)-C(17) & 111.7(4) \\ C(18)-C(19)-C(14) & 112.3(3) \\ C(25)-C(20)-C(21) & 109.4(3) \\ C(25)-C(20)-P(1) & 110.9(3) \\ C(21)-C(20)-P(1) & 119.9(3) \\ C(22)-C(21)-C(20) & 111.6(4) \\ C(23)-C(22)-C(21) & 111.9(4) \\ C(22)-C(23)-C(24) & 110.8(3) \\ C(25)-C(24)-C(23) & 110.1(4) \\ C(24)-C(25)-C(20) & 111.5(3) \\ C(31)-C(26)-C(27) & 111.1(3) \\ C(31)-C(26)-P(2) & 117.8(2) \\ C(27)-C(26)-P(2) & 113.6(2) \\ C(28)-C(27)-C(26) & 111.0(3) \\ C(29)-C(28)-C(27) & 110.8(3) \\ C(30)-C(29)-C(28) & 111.3(3) \\ C(29)-C(30)-C(31) & 111.0(3) \\ C(30)-C(31)-C(26) & 110.7(3) \\ C(33)-C(32)-C(37) & 110.0(3) \\ C(33)-C(32)-P(2) & 110.7(2) \\ C(37)-C(32)-P(2) & 119.1(3) \\ C(32)-C(33)-C(34) & 111.9(3) \\ C(35)-C(34)-C(33) & 110.3(4) \\ C(36)-C(35)-C(34) & 110.0(3) \\ C(35)-C(36)-C(37) & 112.5(3) \\ C(36)-C(37)-C(32) & 110.9(3) \\ C(43)-C(38)-C(39) & 110.1(3) \\ C(43)-C(38)-P(2) & 109.9(3) \\ C(39)-C(38)-P(2) & 115.0(3) \\ C(40)-C(39)-C(38) & 111.6(3) \\ C(39)-C(40)-C(41) & 111.9(4) \\ C(42)-C(41)-C(40) & 110.6(3) \\ C(41)-C(42)-C(43) & 111.3(4) \\ C(42)-C(43)-C(38) & 112.2(3) \\ C(44)-C(45)-C(46) & 131.3(14) \\ C(47)-C(46)-C(45) & 107.9(15) \\ C(46)-C(47)-C(48) & 105.1(16) \\ & \\ C & \end{array}$


Table S4. Anisotropic displacement parameters $\left(A^{\wedge} 2 \times 10^{\wedge} 3\right)$ for Compound 2 . (pentane). The anisotropic displacement factor exponent takes the form:

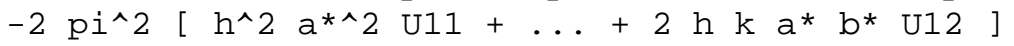

\begin{tabular}{|c|c|c|c|c|c|c|}
\hline & U11 & U22 & U33 & U23 & U13 & U12 \\
\hline $\mathrm{Ni}(1)$ & $24(1)$ & $25(1)$ & $26(1)$ & $-4(1)$ & $13(1)$ & $-4(1)$ \\
\hline$P(1)^{\prime}$ & $25(1)$ & $25(1)$ & $25(1)$ & $-1(1)$ & $12(1)$ & $1(1)$ \\
\hline$P(2)$ & $21(1)$ & $23(1)$ & $25(1)$ & $-1(1)$ & $9(1)$ & $0(1)$ \\
\hline$C(1)$ & $64(3)$ & $51(2)$ & $56(3)$ & $-26(2)$ & $45(3)$ & $-34(2)$ \\
\hline$C(2)$ & $87(4)$ & $55(3)$ & $48(3)$ & $-22(2)$ & $51(3)$ & $-37(2)$ \\
\hline$C(3)$ & $124(5)$ & $73(3)$ & $85(4)$ & $-4 \odot(3)$ & $85(4)$ & $-55(3)$ \\
\hline$C(4)$ & $45(3)$ & $36(2)$ & $112(5)$ & $-4(2)$ & $45(3)$ & $-1(2)$ \\
\hline$c(5)$ & $50(3)$ & $69(3)$ & $69(4)$ & $11(3)$ & $25(3)$ & $\odot(2)$ \\
\hline$C(6)$ & $103(5)$ & $78(4)$ & $59(4)$ & $27(3)$ & $53(4)$ & $46(3)$ \\
\hline$c(7)$ & $93(4)$ & $48(3)$ & $60(3)$ & $-20(2)$ & $51(3)$ & $-38(2)$ \\
\hline$C(8)$ & $29(2)$ & $28(2)$ & $30(2)$ & $-1(1)$ & $14(2)$ & $3(1)$ \\
\hline$C(9)$ & $35(2)$ & $34(2)$ & $33(2)$ & $1(2)$ & $12(2)$ & $7(1)$ \\
\hline$C(10)$ & $44(2)$ & $34(2)$ & $45(2)$ & $6(2)$ & $16(2)$ & $12(2)$ \\
\hline$c(11)$ & $56(3)$ & $37(2)$ & $58(3)$ & $-2(2)$ & $24(2)$ & $17(2)$ \\
\hline$C(12)$ & $63(3)$ & $34(2)$ & $47(3)$ & $-8(2)$ & $24(2)$ & $8(2)$ \\
\hline$C(13)$ & $46(2)$ & $34(2)$ & $36(2)$ & $\odot(2)$ & $20(2)$ & $9(2)$ \\
\hline$c(14)$ & $27(2)$ & $32(2)$ & $23(2)$ & $2(1)$ & $11(1)$ & $0(1)$ \\
\hline$C(15)$ & $34(2)$ & $40(2)$ & $34(2)$ & $6(2)$ & $3(2)$ & $-10(2)$ \\
\hline$C(16)$ & $36(2)$ & $49(2)$ & $38(2)$ & $9(2)$ & $3(2)$ & $-8(2)$ \\
\hline$C(17)$ & $28(2)$ & $69(3)$ & $49(3)$ & $3(2)$ & $6(2)$ & $-1(2)$ \\
\hline$C(18)$ & $37(2)$ & $43(2)$ & $58(3)$ & $5(2)$ & $\odot(2)$ & $8(2)$ \\
\hline$C(19)$ & $28(2)$ & $36(2)$ & $44(2)$ & $3(2)$ & $6(2)$ & $7(2)$ \\
\hline$C(20)$ & $30(2)$ & $27(2)$ & $37(2)$ & $2(1)$ & $19(2)$ & $1(1)$ \\
\hline$C(21)$ & $54(3)$ & $46(2)$ & $38(2)$ & $7(2)$ & $30(2)$ & $-2(2)$ \\
\hline$c(22)$ & $64(3)$ & $51(2)$ & $53(3)$ & $10(2)$ & $40(3)$ & $-2(2)$ \\
\hline$C(23)$ & $53(3)$ & $44(2)$ & $82(4)$ & $8(2)$ & $49(3)$ & $2(2)$ \\
\hline$C(24)$ & $31(2)$ & $43(2)$ & $69(3)$ & $6(2)$ & $27(2)$ & $1(2)$ \\
\hline$c(25)$ & $29(2)$ & $33(2)$ & $49(2)$ & $3(2)$ & $20(2)$ & $3(1)$ \\
\hline$C(26)$ & $24(2)$ & $29(2)$ & $28(2)$ & $\odot(1)$ & $12(1)$ & $3(1)$ \\
\hline$C(27)$ & $24(2)$ & $36(2)$ & $34(2)$ & $-3(2)$ & $11(2)$ & $1(1)$ \\
\hline$c(28)$ & $27(2)$ & $47(2)$ & $47(2)$ & $6(2)$ & $19(2)$ & $7(2)$ \\
\hline C(29) & $43(2)$ & $46(2)$ & $52(3)$ & $4(2)$ & $32(2)$ & $16(2)$ \\
\hline$C(30)$ & $52(2)$ & $37(2)$ & $40(2)$ & $-4(2)$ & $24(2)$ & $9(2)$ \\
\hline$c(31)$ & $39(2)$ & $28(2)$ & $34(2)$ & $-4(1)$ & $16(2)$ & $4(1)$ \\
\hline$C(32)$ & $24(2)$ & $28(2)$ & $30(2)$ & $1(1)$ & $12(2)$ & $-1(1)$ \\
\hline$C(33)$ & $31(2)$ & $28(2)$ & $30(2)$ & $3(1)$ & $11(2)$ & $\Theta(1)$ \\
\hline$c(34)$ & $44(2)$ & $43(2)$ & $42(2)$ & $12(2)$ & $20(2)$ & $2(2)$ \\
\hline$C(35)$ & $53(3)$ & $40(2)$ & $61(3)$ & $11(2)$ & $28(2)$ & $-6(2)$ \\
\hline$C(36)$ & $43(2)$ & $38(2)$ & $55(3)$ & $-1(2)$ & $22(2)$ & $-16(2)$ \\
\hline$c(37)$ & $29(2)$ & $32(2)$ & $42(2)$ & $-1(2)$ & $10(2)$ & $-9(1)$ \\
\hline$C(38)$ & $24(2)$ & $27(2)$ & $29(2)$ & $\odot(1)$ & $8(2)$ & $\Theta(1)$ \\
\hline$C(39)$ & $33(2)$ & $41(2)$ & $28(2)$ & $3(2)$ & $9(2)$ & $-1(2)$ \\
\hline$C(40)$ & $44(2)$ & $44(2)$ & $25(2)$ & $5(2)$ & $6(2)$ & $2(2)$ \\
\hline$C(41)$ & $42(2)$ & $53(3)$ & $46(3)$ & $13(2)$ & $6(2)$ & $16(2)$ \\
\hline$C(42)$ & $29(2)$ & $77(3)$ & $46(3)$ & $3(2)$ & $5(2)$ & $14(2)$ \\
\hline$C(43)$ & $26(2)$ & $47(2)$ & $39(2)$ & $2(2)$ & $10(2)$ & $9(2)$ \\
\hline$C(44)$ & $300(20)$ & $248(16)$ & $108(9)$ & $-49(10)$ & $75(11)$ & $-162(16)$ \\
\hline$c(45)$ & $620(50)$ & $250(20)$ & $500(40)$ & $150(20)$ & $490(40)$ & $60(20)$ \\
\hline
\end{tabular}




$\begin{array}{llllrrr}C(46) & 174(12) & 330(20) & 108(9) & 127(12) & 22(9) & -67(12) \\ C(47) & 198(13) & 192(13) & 125(10) & 66(9) & -3(10) & -99(11) \\ C(48) & 420(30) & 143(12) & 620(40) & 94(17) & 440(30) & 79(14)\end{array}$

Table S5. Hydrogen coordinates ( $x$ 10^4) and isotropic displacement parameters $\left(A^{\wedge} 2 \times 10 \wedge 3\right)$ for Compound $2 \cdot($ pentane).

\begin{tabular}{|c|c|c|c|c|}
\hline & $x$ & $y$ & $z$ & $\mathrm{U}(\mathrm{eq})$ \\
\hline$H(2 A)$ & 1841 & 2682 & 4322 & 67 \\
\hline$H(3 A)$ & 1032 & 2998 & 4955 & 96 \\
\hline$H(3 B)$ & 466 & 3652 & 4271 & 96 \\
\hline$H(4 A)$ & -375 & 2397 & $4 \odot 52$ & 72 \\
\hline$H(5 A)$ & -363 & 2407 & 2622 & 75 \\
\hline$H(5 B)$ & -140 & 3353 & 2967 & 75 \\
\hline$H(6 A)$ & 146 & 1147 & 3777 & 89 \\
\hline$H(6 B)$ & 1012 & 1479 & 4467 & 89 \\
\hline$H(7 A)$ & 578 & 1382 & 2821 & 72 \\
\hline$H(7 B)$ & $15 \odot 7$ & 1462 & 3542 & 72 \\
\hline$H(8 A)$ & 1468 & 1860 & 2028 & 34 \\
\hline$H(9 A)$ & 3214 & 2189 & 2804 & 41 \\
\hline$H(9 B)$ & 2623 & 2142 & 3261 & 41 \\
\hline $\mathrm{H}(10 \mathrm{~A})$ & 3294 & 828 & 3328 & 51 \\
\hline$H(10 B)$ & 2297 & 732 & 2880 & 51 \\
\hline$H(11 A)$ & 3397 & 815 & 2105 & 61 \\
\hline$H(11 B)$ & 2873 & 7 & 2133 & 61 \\
\hline$H(12 A)$ & 1616 & 655 & 1312 & 58 \\
\hline $\mathrm{H}(12 \mathrm{~B})$ & 2228 & 701 & 878 & 58 \\
\hline$H(13 A)$ & 2555 & 2113 & 1278 & 45 \\
\hline$H(13 B)$ & 1559 & 2018 & 797 & 45 \\
\hline$H(14 A)$ & 702 & 3291 & 626 & 33 \\
\hline$H(15 B)$ & 251 & 2073 & 1018 & 48 \\
\hline$H(15 C)$ & -76 & 2594 & 1568 & 48 \\
\hline $\mathrm{H}(16 \mathrm{~A})$ & -786 & 2659 & -146 & 55 \\
\hline $\mathrm{H}(16 \mathrm{~B})$ & -1229 & 2256 & 366 & 55 \\
\hline$H(17 A)$ & -1379 & 3576 & 832 & 62 \\
\hline $\mathrm{H}(17 \mathrm{~B})$ & -1795 & 3585 & -128 & 62 \\
\hline$H(18 A)$ & $-6 \odot 7$ & 4243 & -130 & 64 \\
\hline $\mathrm{H}(18 \mathrm{~B})$ & -937 & 4790 & $4 \odot 2$ & 64 \\
\hline$H(19 A)$ & 110 & 4256 & 1584 & 47 \\
\hline$H(19 B)$ & 544 & 4627 & 1051 & 47 \\
\hline$H(2 \odot A)$ & 2126 & 4556 & 1727 & 36 \\
\hline$H(21 A)$ & 2377 & 3327 & 736 & 51 \\
\hline$H(21 B)$ & 1612 & 3975 & 437 & 51 \\
\hline$H(22 A)$ & 2701 & 4455 & 104 & 61 \\
\hline$H(22 B)$ & 2532 & 5110 & 674 & 61 \\
\hline$H(23 A)$ & 3975 & 4938 & 1139 & 63 \\
\hline$H(23 B)$ & 3887 & 3944 & 1182 & 63 \\
\hline$H(24 A)$ & 4338 & 4499 & 2489 & 55 \\
\hline$H(24 B)$ & 3558 & 5130 & 2187 & 55 \\
\hline
\end{tabular}




\begin{tabular}{|c|c|c|c|c|}
\hline$H(25 A)$ & 3268 & 3965 & 2822 & 43 \\
\hline$H(25 B)$ & 3425 & 3338 & 2224 & 43 \\
\hline$H(26 A)$ & 1259 & 4880 & 4434 & 32 \\
\hline$H(27 A)$ & 381 & 5675 & 2927 & 38 \\
\hline$H(27 B)$ & 261 & 4715 & 3105 & 38 \\
\hline$H(28 A)$ & -795 & 5576 & 3211 & 47 \\
\hline$H(28 B)$ & -249 & 5113 & 4042 & 47 \\
\hline$H(29 A)$ & -28 & 6824 & 3691 & 52 \\
\hline$H(29 B)$ & -405 & 6535 & 4300 & 52 \\
\hline$H(3 \odot A)$ & 895 & 5973 & 5175 & 50 \\
\hline$H(3 \odot B)$ & 1019 & 6935 & 5007 & 50 \\
\hline$H(31 A)$ & 2086 & 6080 & 4910 & 40 \\
\hline$H(31 B)$ & 1548 & 6546 & 4078 & 40 \\
\hline$H(32 A)$ & 2737 & 5260 & 2958 & 32 \\
\hline$H(33 A)$ & 1356 & 6305 & 2748 & 36 \\
\hline H(33B) & 1310 & 5520 & 2193 & 36 \\
\hline$H(34 A)$ & 2235 & 6189 & 1752 & 51 \\
\hline$H(34 B)$ & 1447 & 6786 & 1576 & 51 \\
\hline$H(35 A)$ & 2723 & 7559 & 2174 & 60 \\
\hline$H(35 B)$ & 2235 & 7583 & 2732 & 60 \\
\hline$H(36 A)$ & 3655 & 7282 & 3521 & 54 \\
\hline$H(36 B)$ & 3609 & 6500 & 2963 & 54 \\
\hline$H(37 A)$ & 2776 & 6604 & 3993 & 43 \\
\hline$H(37 B)$ & 3552 & 6002 & 4132 & 43 \\
\hline$H(38 A)$ & 3236 & 5241 & 4886 & 34 \\
\hline$H(39 A)$ & 2415 & 4683 & 5489 & 43 \\
\hline$H(39 B)$ & 2556 & 3754 & 5249 & 43 \\
\hline$H(4 \odot A)$ & 3469 & 3982 & 6595 & 49 \\
\hline$H(40 B)$ & 3836 & 4805 & 6366 & 49 \\
\hline$H(41 A)$ & 4788 & $37 \odot 3$ & 6588 & 62 \\
\hline$H(41 B)$ & 4070 & 3130 & 5945 & 62 \\
\hline$H(42 A)$ & 4880 & 3696 & 5338 & 65 \\
\hline$H(42 B)$ & 4734 & 4624 & 5579 & 65 \\
\hline$H(43 A)$ & 3461 & 3578 & 4438 & 47 \\
\hline$H(43 B)$ & 3831 & 4406 & 4223 & 47 \\
\hline$H(44 A)$ & 4154 & 8439 & 2015 & 334 \\
\hline$H(44 B)$ & 3436 & 8352 & 1121 & 334 \\
\hline$H(44 C)$ & 4380 & 8545 & 1269 & 334 \\
\hline$H(45 A)$ & 3628 & 7175 & 1357 & 444 \\
\hline$H(45 B)$ & 4526 & 7316 & 2059 & 444 \\
\hline$H(46 A)$ & 4144 & 6520 & 655 & 262 \\
\hline$H(46 B)$ & 4771 & 7273 & 720 & 262 \\
\hline$H(47 A)$ & 4984 & 6142 & 1877 & 236 \\
\hline$H(47 C)$ & 5621 & 6874 & 1904 & 236 \\
\hline$H(48 C)$ & 5546 & 5370 & 1245 & 482 \\
\hline$H(48 D)$ & 6047 & 6154 & 1130 & 482 \\
\hline$H(48 A)$ & 5103 & 5979 & 493 & 482 \\
\hline
\end{tabular}

